\title{
Editorial
}

\section{Una mirada a las universidades venezolanas a través de los rankings académicos}

Mucho se ha escrito sobre la difícil situación de las instituciones de educación superior (IES) venezolanas, la mayoría lo trata como un problema político-ideológico y consideran para su análisis aspectos como: déficit presupuestario, cese de beneficios profesorales y estudiantiles, deserción estudiantil, baja calidad de la educación, fuga de talentos, inseguridad, carencia de visión y objetivos, entre otros; para continuar aportando a este análisis de la situación de las universidades venezolanas, se debe estudiar el posicionamiento que estas tienen en los rankings universitarios. Los rankings universitarios, son una herramienta de información valiosa que nos da una visión de la calidad y prestigio de las universidades y por ende de los países en su educación superior. Los rankings utilizan diferentes metodologías para comparar la investigación, docencia, impacto, divulgación y administración; de allí la importancia de conocer sus metodologías, pues la información que aportan es valiosa para la toma de decisiones en la planificación, generación o corrección de políticas y cambios organizacionales que coadyuven a mejorar, modernizar y posicionar a las instituciones de educación superior. Entre los más destacados rankings se encuentran:

1. Academic Ranking of World Universities (ARWU), http://www.shanghairanking.com. Analiza a las universidades a través de indicadores que miden la producción e impacto de la investigación, dentro de estos indicadores tenemos premios Nobel, medallas Fields, investigadores altamente citados, artículos publicados en Nature y en Science, artículos indexados en Science Citation Index Expanded (SCIE) y en Social Science Citation Index (SSCI).

2. Times Higher Education World University Rankings (THE), https://www.timeshighereducation.com. Evalúa a las universidades a través de 13 indicadores que miden la docencia, investigación, impacto, internacionalización e ingresos de la industria. Para determinar la cantidad de artículos publicados y su citación, usa la base de datos Scopus.

3. QS World University Rankings (QS), http://www.iu.qs.com. Utiliza una metodología específica para América Latina, conservando los indicadores de la clasificación global. Dentro de los indicadores tenemos: reputación académica, reputación del empleador, proporción profesores a estudiantes, personal con doctorado, publicaciones en colaboración internacional, entre otras. Usa la base de datos Scopus para evaluar el impacto de la investigación.

4. Scimago Institutions Rankings (SIR) https://www.scimagojr.com. Aplica indicadores basados en el rendimiento de la investigación, los resultados de la innovación y el impacto social medido por su visibilidad en la web. Ofrece información por institución, país, evolución histórica, comparativos, entre otros. Para evaluar la investigación, usa la base de datos Scopus.

Ubicación de nuestras IES y la presencia de Venezuela en estos rankings, comparándolos con la América Latina:

Ranking ARWU 2020: ninguna institución venezolana aparece, Brasil lidera con 22 universidades, Chile con 4, Argentina con 3, México con 2, y Colombia, Uruguay y Costa Rica, con 1, respectivamente. Es de hacer notar que la mayoría de las universidades clasifican por el volumen de publicaciones científicas, con las que cuentan en los grupos editoriales y los índices de orden mundial, como Nature, Science, SCIE y SSCI.

Ranking THE: para el año de evaluación se toman en cuenta los artículos indexados en la base de datos Scopus, por un periodo de 5 años, sin incluir el año de evaluación y el año anterior, así para el 2021, se evalúa el periodo 2015-2019. Para aparecer en este ranking, las universidades deben publicar un mínimo de 1.000 trabajos en el periodo evaluado.

- Ranking mundial 2016-2017, periodo evaluado 2011-2015, con 981 universidades evaluadas, la Universidad de Los Andes (ULA) y Universidad Simón Bolívar (USB), se encontraban después de la posición 801.

- Ranking mundial 2018, periodo evaluado 2012-2016, con 1.103 universidades evaluadas, la Universidad Central de Venezuela (UCV) y USB aparecen entre las posiciones 801-1.000, y ULA después de la posición 1.001.

- Ranking mundial 2019, periodo evaluado 2013-2017, con 1.258 universidades evaluadas, ULA y USB aparecen después de la posición 1.001 .

- Ranking mundial 2020, periodo evaluado 2014-2018, con 1.397 universidades evaluadas, ULA y la USB aparecen después de la posición 1.001 .

- Ranking mundial 2021, periodo evaluado 2015-2019, con 1.526 universidades evaluadas, ULA y USB aparecen después de la posición 1.001 . 
- Ranking latinoamericano 2016, con 50 universidades evaluadas, USB entre las posiciones 31-35 y ULA entre 4145.

- Ranking latinoamericano 2017, con 82 universidades evaluadas, USB entre las posiciones 26-30, UCV y ULA entre 46-50.

- Ranking latinoamericano 2018, con 129 universidades evaluadas, USB ocupa la posición 39 y ULA entre las posiciones 61-70.

- Ranking latinoamericano 2019, con 150 universidades evaluadas, USB entre las posiciones 51-60 y ULA entre 6170.

- Ranking latinoamericano 2020, con 166 universidades evaluadas, ULA ocupa la posición 57 y USB la 72.

Ranking QS: a continuación, se muestra el posicionamiento en el ranking mundial y el latinoamericano, la diferencia de posicionamiento se debe al uso de metodologías diferentes para su cálculo.

- Ranking mundial 2018, con 977 universidades evaluadas, Universidad Central de Venezuela (UCV) y Universidad Católica Andrés Bello (UCAB) entre las posiciones 651-700, le siguen USB entre 751-800, por último, ULA y Universidad Metropolitana (UM) entre la posición 801-1000.

- Ranking mundial 2021, con 1.186 universidades evaluadas, UCV entre las posiciones 751-800, ULA, UCAB y USB entre 801-1.000, por último, UM después de la posición 1.001.

- Ranking latinoamericano 2018, con 385 universidades evaluadas, UCV ocupó la posición 28, USB la 39, UCAB la 65, ULA la 75, Universidad del Zulia (LUZ) la 131, UM después de la posición 149 y Universidad de Carabobo entre las posiciones 171-180.

- Ranking latinoamericano 2021, con 410 universidades evaluadas, UCV ocupa la posición 41, USB la 49, ULA la 75, UCAB la 76, LUZ entre 151-160, UM entre 161-170 y UC entre 171-180.

Ranking SIR: para este editorial, ofreceremos los resultados del año 2021; en su sitio Web se consigue la información desde el 2009 hasta el 2021, cabe resaltar que instituciones como Universidad de Carabobo (UC) y Universidad de Oriente (UDO), estuvieron en el ranking en años anteriores, actualmente no califican, pues no cumplen con algunos criterios de la metodología. Las universidades que publiquen menos de 100 artículos relevantes al año son excluidas del ranking.

- Ranking mundial 2021, se evaluaron 4.126 instituciones y para fines comparativos, el valor indicador lo establece en una escala de 0 a 100, así, en el ranking mundial, UCV está en el percentil 58, ULA en el 60, LUZ en el 70 y 1 USB en el 73.

- Por último, el Ranking Scimago, ofrece información y comparaciones de la producción de artículos científicos por países, según sus datos, tomados de la base de datos Scopus, Venezuela en 1997 generó 1.256 artículos científicos citables; en los siguientes años creció hasta alcanzar un máximo de 2.315 artículos citables en 2009; a partir de 2010 comienza a decrecer y para 2019 se publicaron 1.235 artículos citables, 25 menos que los publicados en el año 1997. Como país, Venezuela desde 1996 hasta 2006, generaba más artículos de investigación que Cuba, Colombia, Perú, Ecuador y Uruguay. A partir de 2005, Colombia sobrepasa a Venezuela, lo mismo ocurre con Cuba en el 2010, en el 2014 con Perú, en el 2015 lo hace Ecuador y en el 2017 Uruguay.

Estos datos son preocupantes, pues la caída en la publicación de artículos científicos es sostenida, así como la pérdida de posicionamiento en los rankings, lo que sin lugar a duda nos muestra la decadencia universitaria, cuyo origen es multifactorial; que va desde la imperiosa necesidad de la revisión de la política científica universitaria y gubernamental, de las estructuras anacrónicas tanto del aparato universitario como del Estado, sin dejar de sopesar las incapacidades gerenciales, entre otras, que hoy pudiesen comprometer aún más el ineludible redireccionamiento e impulso que amerita la producción científica en nuestro país.

Nilda Fabiola Rosales López

Universidad de Los Andes, ULA, Venezuela. rfabiola@ula.ve

\footnotetext{
Referencias

Shanghai Ranking Consultancy. Academic Ranking of World Universities. Consultado en http://www.shanghairanking.com.

Times Higher Education, Times Higher Education World University Rankings. Consultado en https://www.timeshighereducation.com.

QS Quacquarelli Symonds. QS World University Rankings. Consultado en http://www.iu.qs.com/.

Scimago Institutions Rankings. Consultado en https://www.scimagojr.com/.
} 